Document downloaded from:

http://hdl.handle.net/10251/85023

This paper must be cited as:

Armesto Ángel, L.; Fuentes-Durá, P.; Perry, DR. (2016). Low-cost Printable Robots in Education. Journal of Intelligent and Robotic Systems. 81(1):5-24. doi:10.1007/s10846-0150199-x.

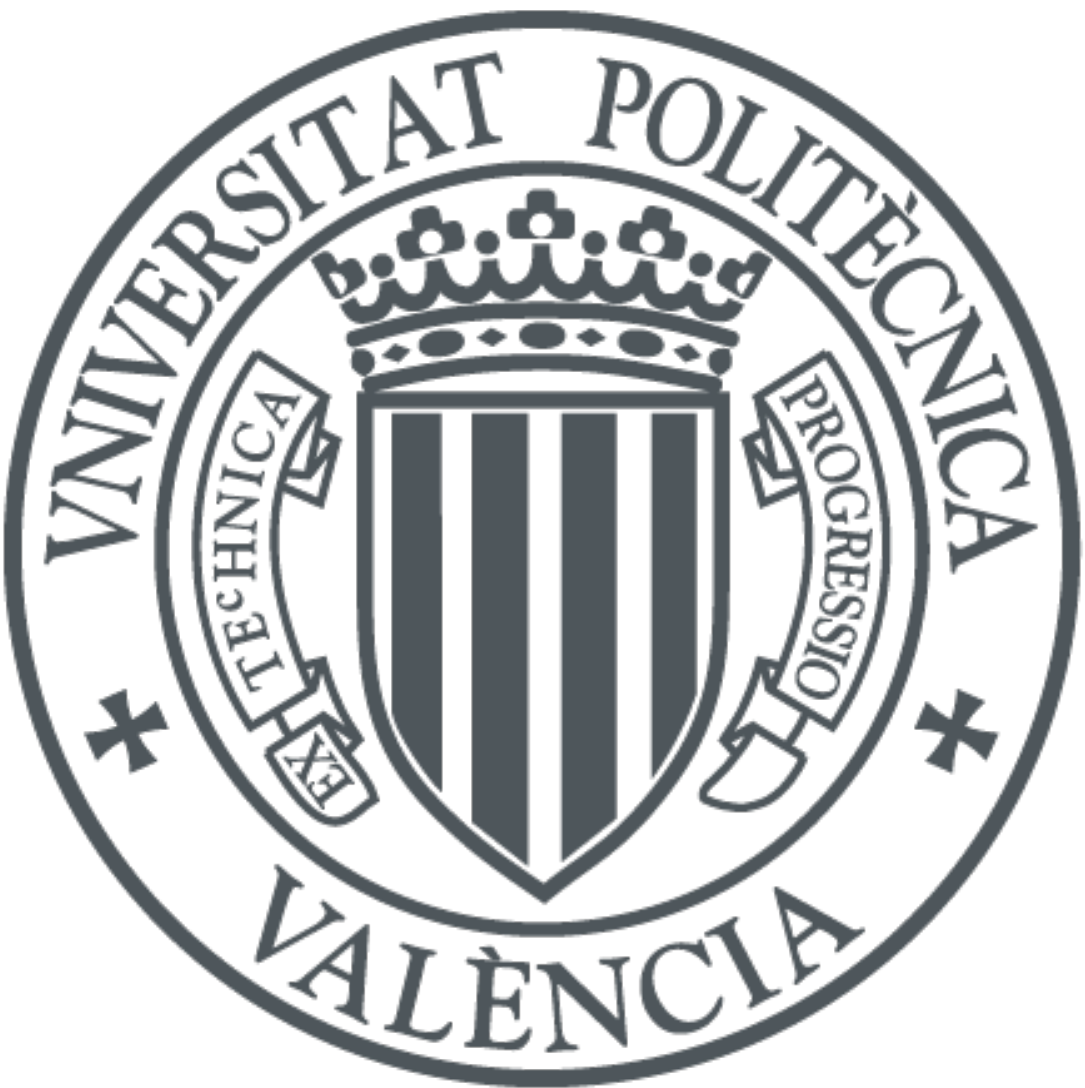

The final publication is available at

http://dx.doi.org/10.1007/s10846-015-0199-x

Copyright Springer Verlag (Germany)

Additional Information

The final publication is available at Springer via http://dx.doi.org/10.1007/s10846-015-0199-x 


\title{
Low-cost Printable Robots in Education
}

\author{
Leopoldo Armesto, Pedro Fuentes-Durá, \\ David Perry
}

Received: date / Accepted: date

\begin{abstract}
The wider availability of 3D printing has enabled small printable robots (or printbots) to be incorporated directly into engineering courses. Printbots can be used in many ways to enhance lifelong learning skills, strengthen understanding and foster teamwork and collaboration. The experiences outlined in this paper were used in our teaching during the last academic year, although much of the methodology and many of the activities have been used and developed over the past 8 years. They include project based assignments carried out by multidisciplinary and multicultural teams, a number of theoretical and practical classroom and laboratory activities all aimed at familiarizing students with fundamental concepts, programming and simulation, and which now form part of our regular robotics courses, and some brief descriptions of how printable robots are being used by students carrying out final projects for Bachelor and Master degrees. The online resources show many of these activities in action.
\end{abstract}

Keywords printbot $\cdot$ robot $\cdot$ education $\cdot 3 \mathrm{D}$ printing

\section{Introduction}

One of the consequences of $3 \mathrm{D}$ printing is that the Robotics Community has the opportunity to reach a much wider public. It is now a relatively straight-

\footnotetext{
Leopoldo Armesto

Instituto de Diseño y Fabricación, Universitat Politécnica de Valéncia, C/Camino de Vera s/n, 46022, Spain, E-mail: larmesto@idf.upv.es

Pedro Fuentes-Durá

Departamento de Ingeniería Química Nuclear, Universitat Politécnica de Valéncia, C/Camino de Vera s/n, 46022, Spain, E-mail: pfuentes@iqn.upv.es

David Perry

Departamento Lingüística Aplicada, Universitat Politécnica de Valéncia, C/Camino de Vera s/n, 46022, Spain, E-mail: daper@idm.upv.es
} 


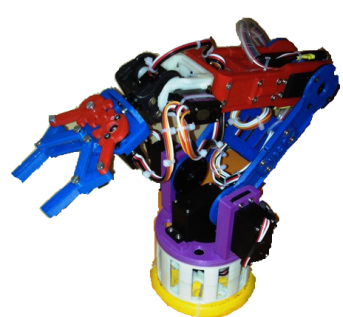

(a) Robot Arm

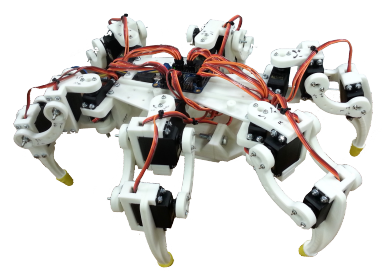

(b) HF08 hexapod

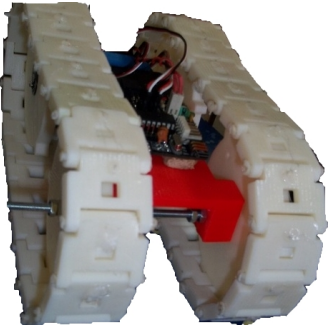

(c) Caterpillator 1.1

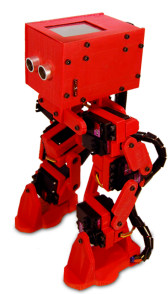

(d) Robot

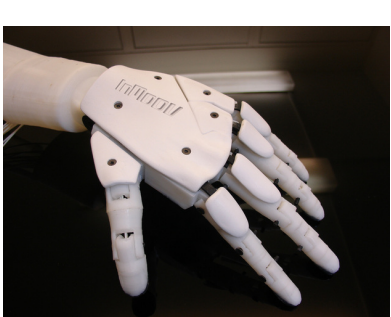

(g) Inmoov hand

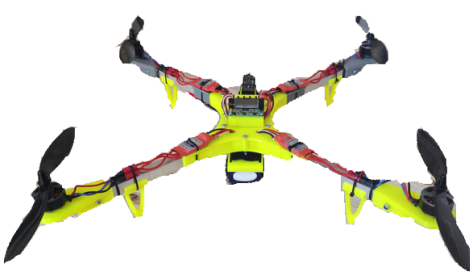

(e) Quadcopter Hummingbird II

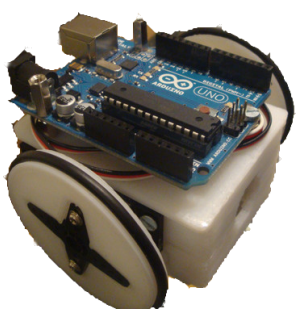

(h) Miniskybot 2

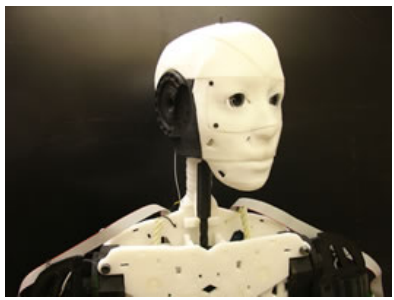

(f) Inmoov head

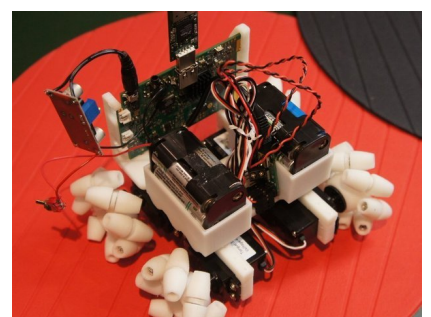

(i) Mecanum Wheel Rover 2

Fig. 1 Examples of free printable robots. From left to right and top to bottom: Robot Arm [5], Hellium From HF08 Hexapod Robot [26], Caterpillator 1.1 [24], ROFI Robot Five [9], Quadcopter Hummingbird II [19], Inmoov head and hand [20], Miniskybot2 [14], Mecanum Wheel Rover 2 [21].

forward process to download many types of free printable robot models, also known as printbots (see Figure 1 for examples), which can be used for research, as well as for other educational and non-commercial purposes. These small robots are far more than simple toys; they can be used in various ways as powerful educational tools for engineering studies. By incorporating them directly into conventional robotics courses and projects, they can be used as teaching aids to improve and develop students' robotics skills; for example, to learn the fundamentals of robotics; to adapt existing designs; to redesign new parts to enable additional sensing capabilities; to design advanced robot controllers for improving stability in walking robots or unmanned aerial vehicles to name a few. 
This paper explores some of the uses of low-cost printable robots for teaching and disseminating robotics, mainly at the higher education level. In the first of two case studies, we describe a project assignment completed by a multidisciplinary team of students attending an innovative educational program called European Project Semester (EPS), while a second case study reports on the use of low-cost printable robots on two regular Robotics courses (Mobile Robotics and Manipulators). We also include descriptions of some of our students' current final projects for Bachelor and Master degrees. Both of the regular courses as well as the European Project Semester are given at the School of Design Engineering and at the School of Industrial Engineering at the UPV. The main difference between the regular robotics courses with respect to the EPS program is that in the former students attend scheduled practical sessions for learning robotic techniques while in EPS these are learned through extended project work.

All these activities improve students' competences, which is the main thesis hold in this paper. The importance of acquiring competences is replacing the classical content-based learning model. The notion of competence, as well as its taxonomy and evaluation, can be defined in several ways, but in general terms it can be regarded as relevant knowledge and skill applied to the standards of performance expected in the workplace. Competence development implies a holistic immersion of learners regarding the potential professional demands.

Competences describe the outcomes of a syllabus in an integral sense, including the mindset, knowledge and skills acquired in the learning period. In the rapidly changing modern socioeconomic context, it could be argued that much of what is relevant and current now will soon be out-of-date and old hat, and for this reason transversal competences such as adaptability and self-learning have become fundamental skills. Other representative transversal competencies are team work, leadership, project work, problem solving, autonomy and flexibility.

Despite the desirability of transversal competences in academic fields and in the labor market, they are not always explicitly established in higher education. This may be due to the difficulty of assessing individual differences such as mindset, study methods or work habits, all of which are capable of generating an infinite number of valid performances.

A number of studies have proposed competences for engineers; the European research survey Careers after Higher Education [30], the ABET criteria [1] and the Tuning project [16] are examples. In Spain, the basic competencies and skills required by an engineer are regulated by law (for an Industrial Engineer, for example, they are regulated by a Spanish Ministerial Order CIN/351/2009 dated 9th February, 2009).

\section{Methodology}

We report on projects carried out during the academic year 2013-2014, and which in some cases form part of larger, ongoing projects. All the work des- 
cribed, however, is based on the teaching methods and practices we have been (and still are) applying, modifying and refining over the past eight years. Our aim is to show the potential benefits of using such approaches in robot education. We hope that the paper will serve for further debate and that the ideas and practices expressed here will be considered as plausible educational alternatives in the field of Robotics. It is possible, of course, that certain specific aspects which are used in our regular courses may not fully fit with other university syllabi in this area but, as academics, our perception is that in the field of educational robotics the use of printbots will soon become standard practice in homes, colleges and universities. The huge advantage offered by the kind of activities we describe here is the greatly increased motivation felt by students, who enthusiastically apply the learning tools and procedures needed in robotics to their projects. In our opinion, with appropriate adaptation of program and contents these kinds of ideas can be applied at many educational levels.

Classical approaches to teach robotics include formalism and maths related with mechanical systems, control systems engineering, informatics and electronics. The methods introduced here in regular Robotic courses and described in detail in Section 4, pretend to work with competencies such as applied thinking, problem solving, comprehension and integration and creativity or innovation. The tools have been designed so that students learn by themselves starting with tutorized materials and solving a set of problem of specific domains that give them confidence, experience and skills to solve more complex problems while getting involved in a highly motivated course in which they can "see" how complex concepts turn into real solutions using their own printed robots or simulations. To understand the experience with printbots on robotics courses, we used a non-experimental single group research design for process evaluations, when the primary purpose is to describe participants' experiences. We implemented the new tools and tasks; we observed the experience over the semester and collected questionnaries to students. That serves us as a pilot reseach, and helps us to identify important variables involved.

The EPS course, described in the next section, aims at enhancing the employability of students by bridging the gap between higher education and the needs of the workplace. EPS is designed to improve transversal skills, in particular multi-cultural tolerance, teamwork and project management. The course formalises the experience of working together in multi-cultural and multidisciplinary teams from different countries and different disciplines. There is a strong focus on the need to develop personal competences, especially the ability to work and communicate within cross-cultural groups. Recently, we published our own list of competences derived from research into students' job preferences [13]. To understand how beliefs were fostered and whether educational benefits were achieved in EPS, we followed the recommendations made by Miles and Huberman [22] for qualitative data analysis, and adopted a collaborative social causal approach. The data collected was coded and clustered into processes, events, and learning elements to facilitate the search for patterns and themes regarding participants' emergent beliefs in their ability 
to innovate. The findings result from an iterative process of moving between inductive and deductive thinking.

\section{Using Printbots in European Project Semester (EPS)}

EPS is a programme offered by 14 European universities in 11 countries throughout Europe to students who have completed at least two years of higher Education. More than 3000 participants from 50 nationalities have now successfully completed the course. EPS is aligned with the $2011 \mathrm{EU}$ modernisation Agenda and Education and Training 2020. EPS corresponds with levels 6 and 7 of the European Qualification Framework [12] and enables students to acquire all the necessary skills and competences in order to deal with the challenges of the changing international environment, become life-long learners able to solve complex and unpredictable professional problems, think across disciplines, be creative in making independent decisions and work in cross-cultural settings.

EPS is based on POL (Project Oriented Learning), PBL (Project-Based Learning), EDBL (Extracurricular Design-Based Learning) and other related methodologies taking the Aalborg model as a reference [29]. Students are required to demonstrate understanding and integration of knowledge in their own specialization and in other broader contexts. The idea behind EPS is for the participants to be immersed in a project in such a complete sense that there conception of what it is broadens over time. Students choose a task, adopt a preferred role, collaborate in the definition of goals and planning, feel part of a team, get the support of the staff and feel the pressure of 360 degrees evaluation. The projects are diverse and a set of options and orientations are available within each particular project. EPS generates a strong learning environment thanks to its multidisciplinary and multicultural atmosphere and the potential of POL supported with teamwork and an open and experimental context. There are activities developed in the class, others in the lab, field activities and the use of many and varied file sharing and communication aids.

Early in the semester EPS students follow a short, intensive course on Planning and Project Management. To work in teams and to lead teams effectively, group project work must be well organized. Students must learn to prepare and chair meetings and to write minutes of the meetings. Supervisors help with technical advice, follow the team process closely, and, if necessary, offer guidance to resolve any problems that may arise, including conflicts. We have devised protocols to enable the participants to become aware of and selfevaluate a number of different aspects of the work involved. These include the quantity and quality of their work, the initiative and cooperation shown, planning, and attitude. Another important aim of an EPS course is to develop communication skills - in English - in a variety of mainly academic and work-related situations and contexts (verbal, written, formal and informal, interpersonal and group). We also explore basic skills such as listening, speaking, questioning and sharing feedback, as well as organising and presenting information in a structured and informative way, through a variety of practical 
activities. Finally, we examine how techniques of persuasion (such as those used in advertising) can be applied to presentations.

Every EPS course includes several phases: course definition, project statements, team creation, brainstorming and planning, task definition, mid-term project development, mid-term project evaluation, project development, project evaluation, exploitation of results.

It is widely recognized that POL fosters divergent thinking and creative thinking. POL also promotes acquisition of self-learning, communication and practical skills. Finding and setting up appropriate projects is not a simple task. Additionally, it requires human, financial and material resources [17] which are, unfortunately, becoming increasingly limited. Robotics projects may stimulate development of creative and system thinking, acquisition of a polytechnic background and practical skills. The participation of students helps to develop skills such as creativity, teamwork, designing and problem solving. Motivation is improved when real world objects are included and robotics provide an interesting and stimulating context for demonstrating and resolving engineering problems [27]. The nature of robotics provides an excellent design experience of an integrated system that includes mechanics, electronics, computation and control. Thanks to robots, the students receive strong, visceral, fun feedback from physically experiencing their work. There is a wide design space for students to explore, generate hypotheses about how things work, and conduct experiments to validate their beliefs and assumptions [35]. The process of selecting, using and experimenting with a robot system for learning offers several pedagogical benefits including [32]:

- The skills needed in robotics can be applied in many professional fields.

- Working with a robot system enables students to apply (and learn) knowledge from several technical fields.

- Carrying out and implementing the robotics projects requires drawing on several scientific methods.

- Human versus artificial intelligence or man-robot interaction can promote the establishment of links between science-technology, education and the humanities.

\subsection{The Hexapod Team}

In this section, we describe the experience and results obtained by a group of EPS students working with printed robots during the academic year 2013/2014. In this case, the team consisted of seven students of different nationalities and from varying academic backgrounds (Mechanical Engineering, Product Design, Electrical/Electronic Engineering and Computer Science). Their task was to create a fictitious company commercializing low-cost printable robots with printed parts and a set of associated software tools. Due to the multidisciplinary nature of the team, the students were able to complete the whole range of tasks shown in the workflow diagram in Figure 2, and which included market research, CAD, 3D printing, robot simulation and robot assembly and 


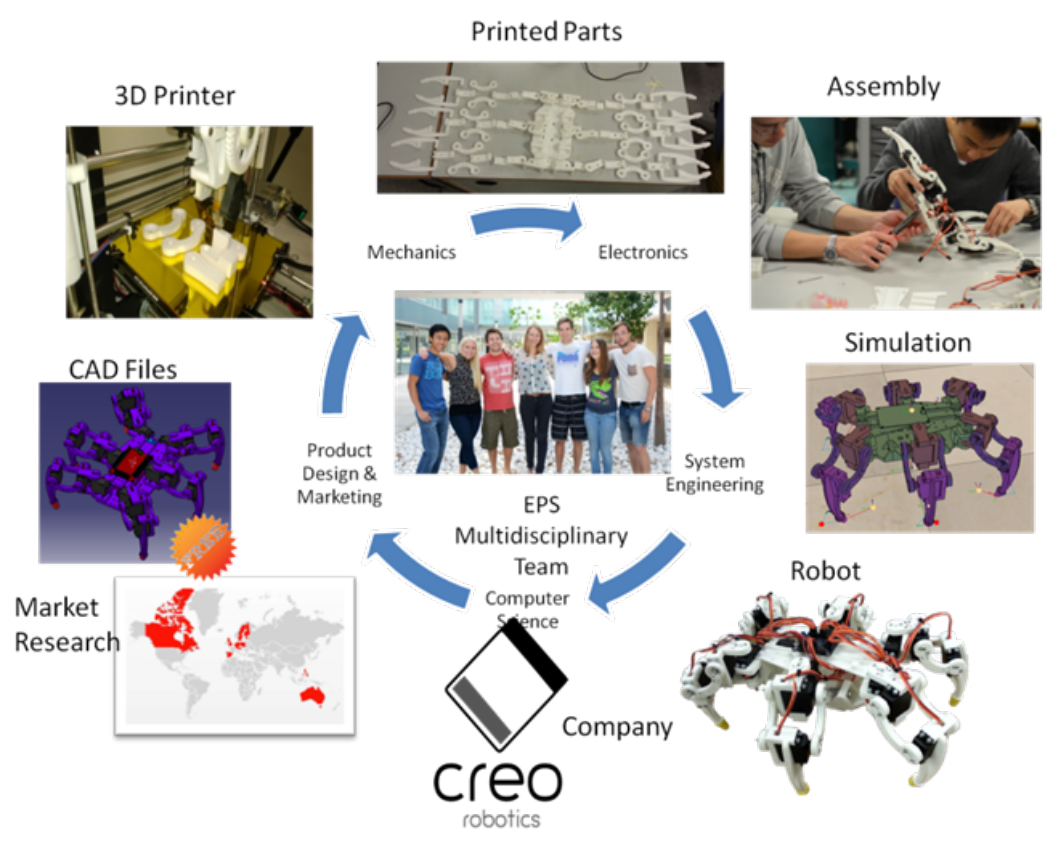

Fig. 2 Workflow of the EPS multidisciplinary team assignment.

control of electronic devices. A logo and a promotional video for the fictitious company were also created. These are shown in Online Resource Material 1.

After carrying out their preliminary market research, the students decided that the first product of their fictitious company would be the Hellium Frog hexapod robot [26]. To reach this decision, they had to analyse all available printable robots and decide which robot would be the best to start with, taking into account aspects such as complexity of parts, available documentation, etc. The parts were $3 \mathrm{D}$ printed, adaptating original CAD files because some of the electronic components such as servos and their controller were different from the original concept. Assembling, calibration and locomotion were also part of the team's tasks.

In addition to this, the team was encouraged to use V-REP in order to simulate robot walking modes before validation with the real robot. This procedure is also described in Section 4. All 3D parts were imported into the simulator, joints were created between each leg link (coxa, femur and tibia) and the robot body (in total, eighteen joints, three per leg, were required). Imported 3D parts were used for the visual layer of the simulation as shown in Figure 3(a), which means that they are simply used for rendering purposes, but no dynamic or physical property is considered. To make the robot walk, collidable objects were created with dynamic properties; that is, a composition of basic primitives such as cuboids, whose simplicity make them suitable not just for dynamic simulation (consideration of torque, force, mass and inertial 


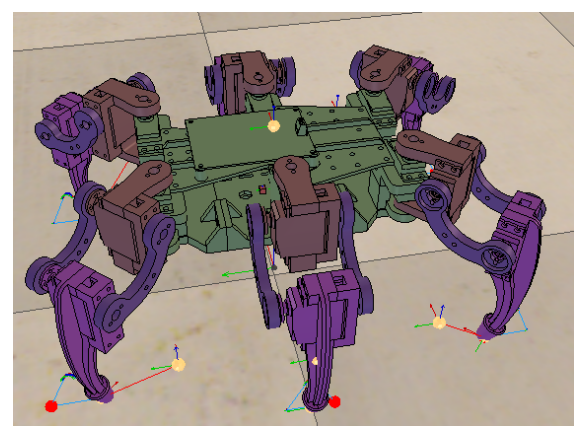

(a) Visual Appearance

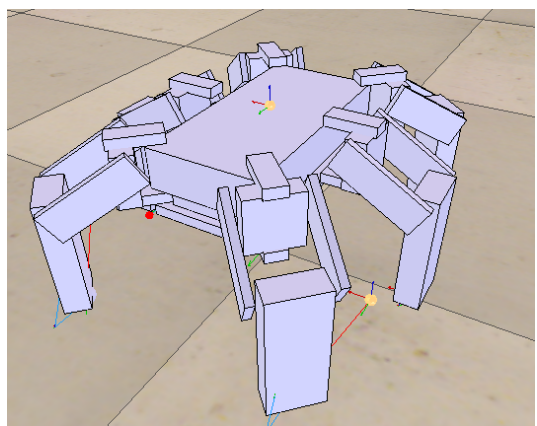

(b) Dynamic and Collidable Objects

Fig. 3 Simulation of HF08 hexapod robot in V-REP.

frames), but also for physical collision checking as shown in Figure 3(b). Robot walking with a regular gait was achieved by creating a two-stage path that the tip of each leg had to follow. Inverse kinematics were solved using V-REP module, which allows students to focus on the gait concept itself rather than the math behind inverse kinematics. Once a gait was validated through simulation, computed angles were transmitted to the real servo angles using a plugin to the servo controller. Despite the fact that the approach uses an open-loop control architecture, it allows the implementation of some rather spectacular robot walking techniques with very few lines of code.

\section{Regular Robotics Courses}

The use of printbots in the classroom also provides many of the advantages offered by POL, and in this section we report on how they have been used in two regular courses. In both, students must complete a set of exercises to understand the fundamentals of robotics through simulations of a printable robot that they can later print, assemble and experiment with. The first course is on Mobile Robotics which uses a Miniskybot 2 [14] as course material. The second course is on Manipulators and uses a robot arm with 6 DOF [5].

\subsection{Mobile Robotics Course}

The course on Mobile Robotics is given during the 4th year of the Bachelor degree in Industrial Electronic and Automation Engineering at the School of Design and at the School of Industrial Engineering at UPV. This course developed out of a previous one given prior to the implantation of the Bologna Process. Students attending the Mobile Robotics course generally have little actual experience of mobile robotics, so the aim is to provide an introduction to the basic fundamentals, including topics such as sensors, actuators, object detection, robot applications, kinematic control, path planning and obstacle 


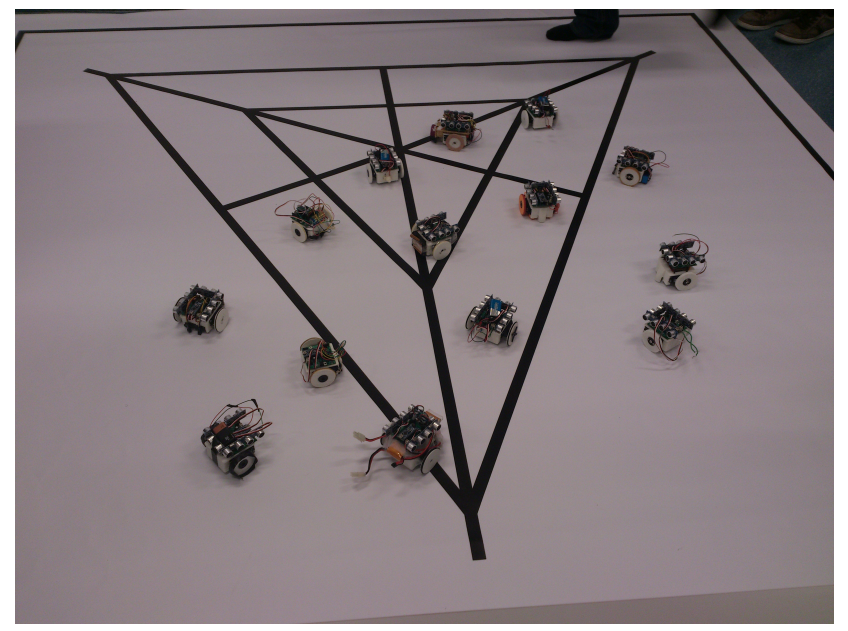

Fig. 4 Printed Miniskybot 2 robots used in lab. sessions

avoidance. The course is mainly targeted on wheeled mobile robots, and theoretical concepts are reinforced with practical exercises using a simulated robot with V-REP robot software simulator [28].

In addition to this, students are required to complete a project assignment with Miniskybot 2 robot [15], in which they must implement some of the ideas they have learned throughout the course, bearing in mind that some of the electronic components have been changed from the original design in order to provide higher sensing capabilities. We have included five ultrasound sensors SF04, one Arduino nano, two CNY70 infrared sensors, two continuous rotational servos SM-S4303R and a marble.

Compared to pre-built existing robots, students are more clearly motivated and involved with this kind of assignment because they feel that they are really building their own robot. Students are encouraged to implement some of the simulated exercises completed previously but this time using the real robot. Figure 4 shows 14 Miniskybot 2 robots used in lab. sessions on an arena mat. Each group of students was responsible for programming, mantaining and tuning its own

Compared to pre-builded existing robots students are more clearly motivated and involved with this kind of assignment because they feel that they are really building their own robot. Students are encouraged to implement some of the previously implemented exercises using the real robot to complete the task assignment. Figure shows 14 miniskybot 2 robots used in Lab. sessions on an arena mat. Each group of students were responsible of, programming, mantaning and tunning their own robot $^{1}$

In our opinion, V-REP is well-suited to educational purposes. Here we describe some of the exercises that students are required to implement with

\footnotetext{
1 STL files, basic instructions and any necessary assistance were given when printing the parts.
} 
this software. These tasks have been designed to improve their competences as described in Section 2. In Online Resource Material 2 we show a summary of exercises and activities carried out by students.

First, students learn to use the simulation environment and get used to it. It is assumed that they have no previous knowledge of the simulation software so preliminary activities are fairly basic being targeted at positioning and rotating some of the Miniskybot 2 components imported directly from CAD files as shown in Figure 5(a). Students are also required to attach joints representing motors, so they need to find the exact position of a servo's axis by editing the servo shape (see Figure 5(b)) and creating and configuring all its dynamic and collidable objects as shown in Figure 5(c). Additional exercises consist of attaching several types of sensors that they will use in further exercises, as well as introducing them to Lua programming and graph representation. Students learn to create their own user interfaces using sliders (see Figure 5(d)), create and configure sonar, attach infrared and vision sensors to the robot as shown in Figure 5(e) and perform their first robot program to move the robot around the environment and measure distance to objects (Figure 5(f)). These preliminary exercises are given in a tutorial-like style, so all students are expected to obtain the same results and to use the same simulated robot for the remaining exercises.

Further exercises are oriented towards learning about some basic obstacle avoidance and control algorithms. For instance, using a front sonar they can implement a proportional controller to regulate the distance to a wall (Figure $5(\mathrm{~g}))$. Another interesting exercise is the implementation of a lateral control algorithm using range sensors (two on each side of the robot) which can be used in corridor-like scenarios. By measuring distances from front-left, back-left, front-right and back-right sensors, the corridor mid-line can be extracted. The pure-pursuit controller [25] is used to converge on the line and, as a result, the angle of a fictitious ${ }^{2}$ front orientable wheel separated by a given distance from the wheel base is computed. Using conventional kinematic relations, left and right wheel velocities can be computed asnd used as set values for motor joints. As a result of this combined reactive obstacle avoidance and kinematic control law, students can easily implement a sensor-based lateral tracking controller with a scenario as shown in Figure 5(h).

At this point, implementation of classic line-following based on infrared sensors (see Figure 5(i)) is also straightforward, where the aim is to stay within the path (if the robot is on the path the sensors detect 'white', while if deviation occurs (at least one of) the sensors will detect 'black'). Here, infrared sensors are, in fact, orthographic cameras with one pixel resolution, so they sense light reflection from the ground. By selecting appropriate threshold values and performing a naive logic to converge to the path, students can easily implement line-followers with just a very few lines of code. The logic simply establishes

2 The robot is a differential robot with no manoeuvrability [3], however we can overcome this aspect by dealing with it as if it were a car-like robot with a fictitious front wheel. 


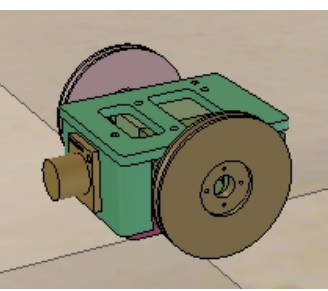

(a) Miniskybot 2

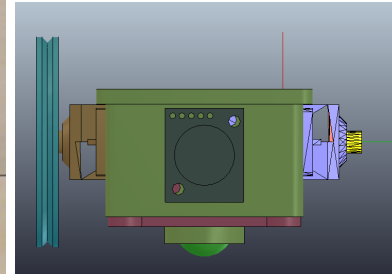

(b) Shape Edition

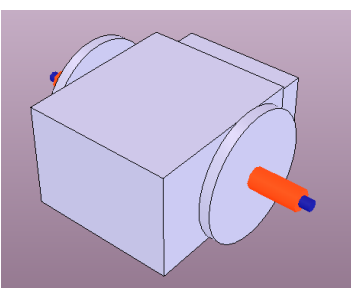

(c) Dynamic Objects

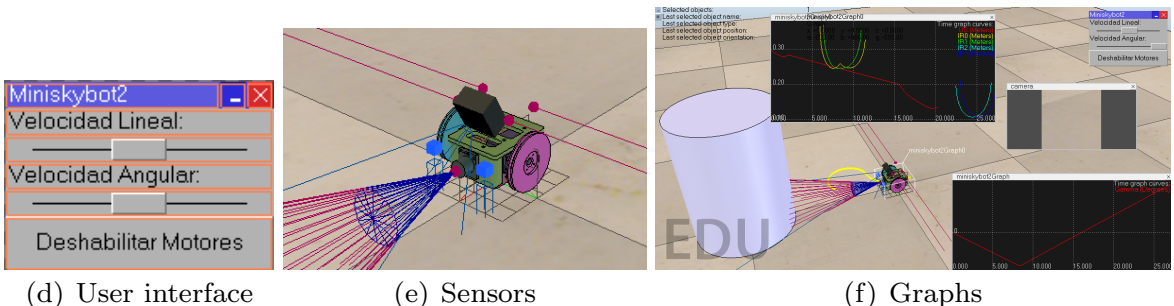

(d) User interface

(e) Sensors

(f) Graphs

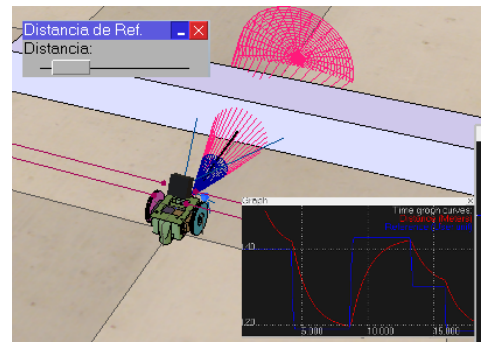

(g) Proportional controller

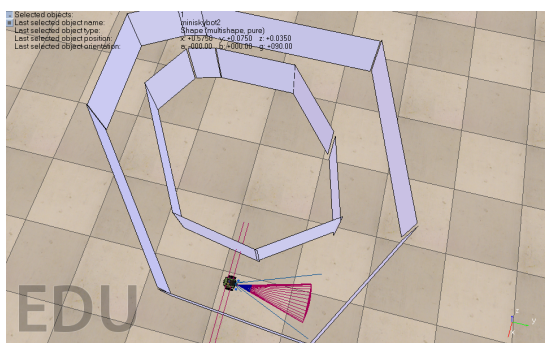

(h) Lateral control

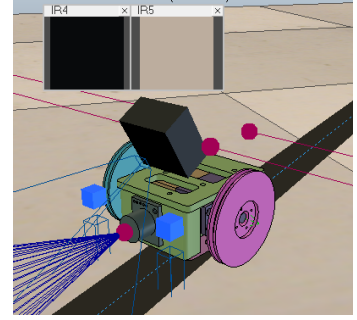

(i) Line-follower

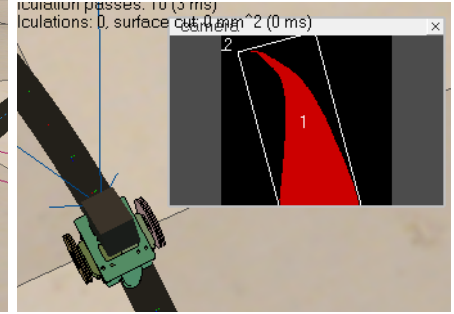

(j) Vision-based line tracking

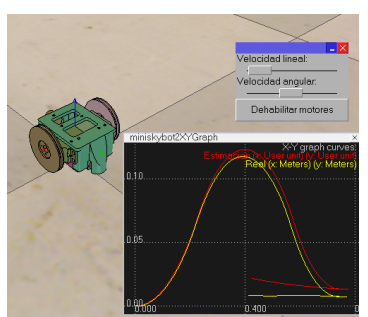

(k) Position estimation

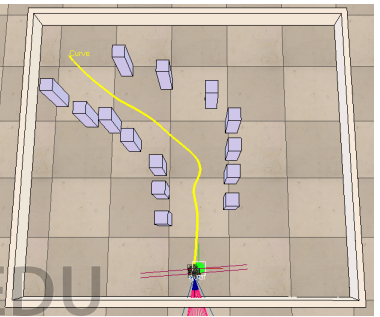

(n) Obstacle avoidance

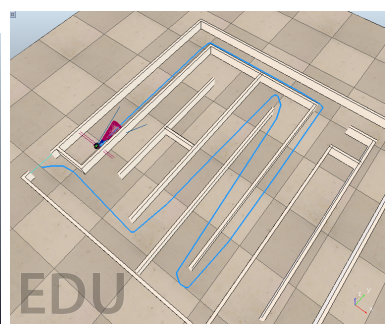

(q) Path planning/Maze solving

Fig. 5 Exercises designed to teach the fundamentals of Mobile Robotics. 
that if a sensor detects the black line, then the velocity of the wheel on the same side is reduced, since the line is expected to be between both sensors.

A more advanced line-following approach can be implemented in V-REP using a vision sensor acting as a perspective camera pointing to the floor with a given pitch angle. The aim of this exercise is to understand some of the fundamentals of traditional computer vision algorithms already implemented in V-REP such as color selection and blob detection. The line can be easily segmented from other objects by obtaining a binary image and then applying a standard blob detection (many small blobs can be found depending on the binarization threshold, but the line is presented as the largest blob). The selected blob provides the horizontal shift of the blob with respect to the image. Line convergence is implemented using pure-pursuit methods, where the aim is to keep the line in the middle of the image.

Trajectory control and path following approaches can also be implemented in V-REP. The former requires defining a path velocity and getting, at each iteration, the trajectory target (depending on the simulation time) and robot positions and orientations. Trajectory control may become unstable if the robot is far from the target point, while path following controllers adapt the target point depending on the current robot position. In both cases, the robot position must be estimated using standard kinematic equations for non-holonomic robots. By implementing exercises, students learn the differences between the real robot position and the estimated one, which inevitably drifts as a consequence of using dead-reckoning sensors, as shown in Figure 5(k). Trajectory control is based on a feed-forward control proposed in [7]. V-REP offers the capability to compute the closest point to a path, which allows us to implement path following controllers. The robot position projected over the path is used to compute a target point for a given distance. Robot trajectory/path control techniques can be used to converge on the path.

A substantially different problem is obstacle avoidance and path planning. Here, Artificial Potential Fields (APF) [18] is used as a representative obstacle avoidance technique. V-REP offers, through its API, the capability for computing distances to obstacles, directly from its sensor readings or by computing distances between objects, which is required by the FIRAS function [18] to implement the Repulsive Potential Field. A combination of Parabolic and Conic functions can be used as Attractive Potential Field for a given goal. By combining them, the robot can navigate the arena shown in Figure 5(n) with very few lines of code. In addition to this, students are also required to implement an algorithm for robot wandering using classical approaches based on a state machine. The idea is to discriminate between cases where the robot has free space to move forward or cases where there is an obstacle just in front of the robot and thus the robot must rotate either left or right depending on sensor readings, see Figure 5(o). Here the purpose is to introduce traditional behavior-based approaches rather than to implement more sophisticated obstacle avoidance methods [23], [31], [11]. Another interesting exercise is the implementation of bug-like algorithms where the robot behaves as if it were 
a bug surrounding obstacles until it crosses the half-plane between the start and goal configurations [6], as shown in Figure 5(p).

Path planning methods can be solved using the "Path Planning" computation module in V-REP. This module requires defining start and goal positions, as well as objects surrounding the robot in order to compute the likelihood of collisions against the remainder of the objects in the scene. For instance, a path can be computed off-line to "safely" navigate through a maze using a cylinder surrounding the robot ${ }^{3}$. Once the path is computed, the goal is to follow the path while also avoiding obstacles. In order to implement this task, students need to draw on some of the concepts they have become familiar with in previous exercises.

\subsection{Robot Manipulator Course}

The course on Robot Manipulators is given in the 4th year of the Mechanical Engineering degree. As with the Mobile Robotics course described above, students attending this course have generally had little experience of robotics, so the aim is to provide an introduction to the basic fundamentals, including geometric representation for positioning and orientation of reference frames, forward and inverse kinematics (particularly focusing on the Denavit-Hartenberg method [8]) and robot programming. Again, theoretical concepts are reinforced with practical exercises using a simulated robot arm with V-REP robot software simulator [28], which is also the one that they print and assemble. This gives students the opportunity to work in teams and learn the fundamentals of robotics from a practical and pragmatic point of view. In other words, they learn concepts by experiencing and experimenting with simulations and also with real robot parts and components.

\subsubsection{Printing and Assembling a Robot Arm}

The course includes lab sessions where two teams (each of six groups of 3-4 students) are responsible for printing and assembling a Robot Arm [5] with $6 \mathrm{DOF}^{4}$. Each group is provided with the list of parts that must be printed in separate printing jobs as shown in Figure 6. The time needed for printing parts for each group is between 2 to 3 hours. In the lab sessions, students are briefly introduced to $3 \mathrm{D}$ printing technology and concepts so they can print the parts themselves, and they usually need to drill some holes or file a few edges due to the poor quality of the printed parts. After that, they need to assemble individually their own link using standard servos, screws, nuts and washers.

\footnotetext{
3 The surrounding cylinder includes a safety margin to avoid the computed path being too close to the walls.

4 As a matter of fact, the robot arm has $7 \mathrm{DOF}$, although two of them act as one to obtain $360^{\circ}$ rotation using two $180^{\circ}$ servos.
} 


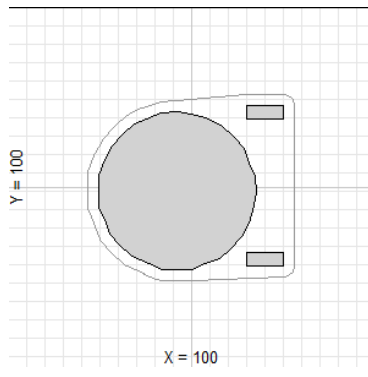

(a) Group 1. Job 1

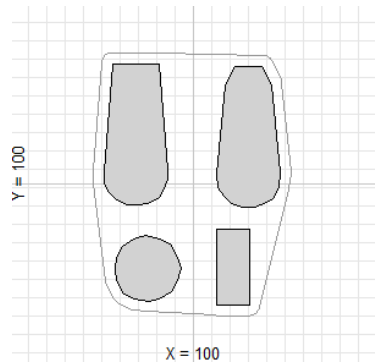

(d) Group 3. Job 1

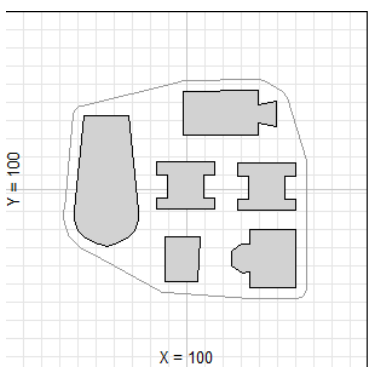

(g) Group 4. Job 2

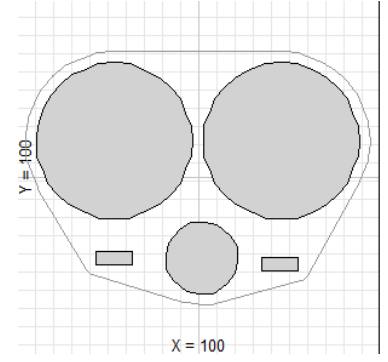

(b) Group 2. Job 1

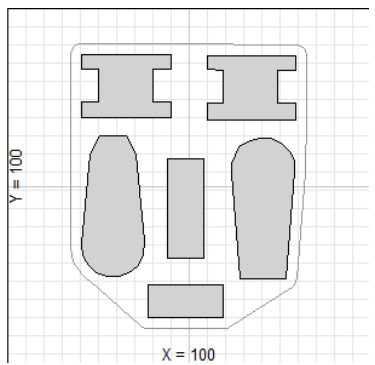

(e) Group 3. Job 2

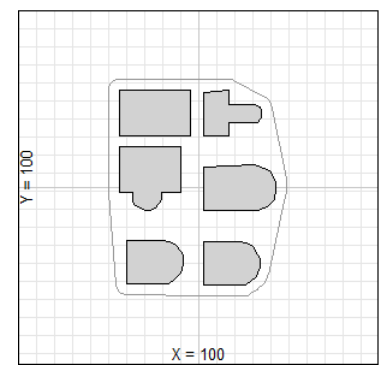

(h) Group 5. Job 1

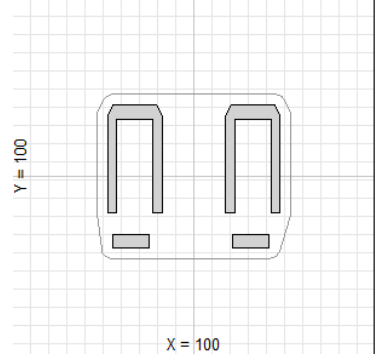

(c) Group 2. Job 2

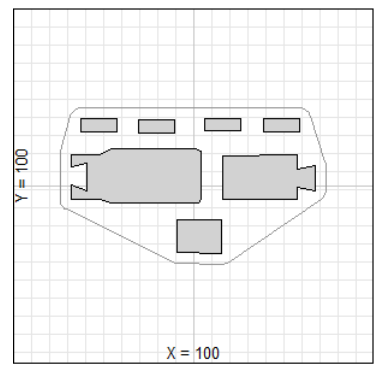

(f) Group 4. Job 1

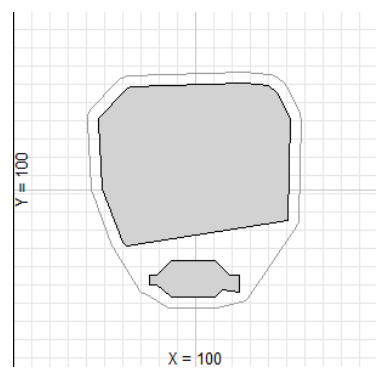

(i) Group 5. Job 2

Fig. 6 Distribution of group assignments and printing jobs.

In the final lab session, all the links for each robot are assembled at the same time. As each group of 3-4 students is responsible for one link, it means that they must coordinate themselves to print link, drill and assemble linked parts and, furthermore, coordinate with the groups responsible for adjacent links.

We have found this to be a highly motivating activity which not only contributes to raising the students' awareness of some of the processes involved in teamwork, but also, of course, strengthens and deepens their understanding of basic concepts such as links and joints, robot design, joint limits and robot workspace, etc., before they start programming and controlling a robot. Figure 7 shows some pictures taken at one of these final assembly sessions. Online Resource Material 3 shows a video with the complete performance. 


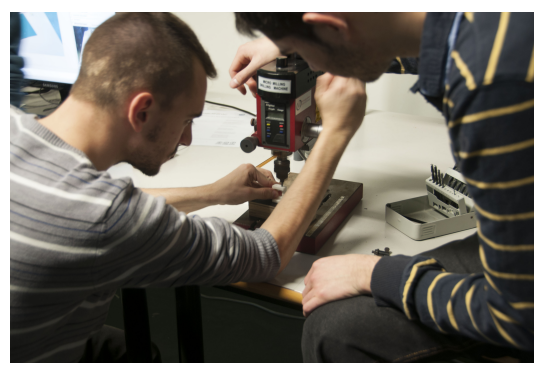

(a) Drilling

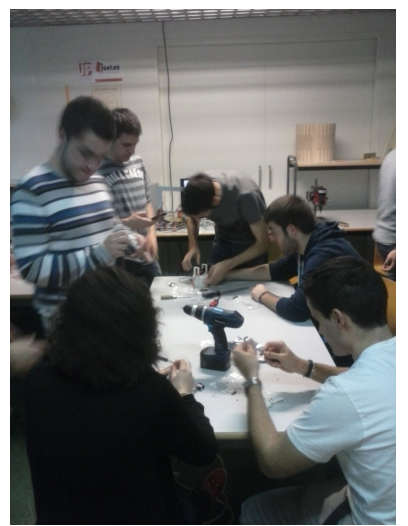

(c) Team 2

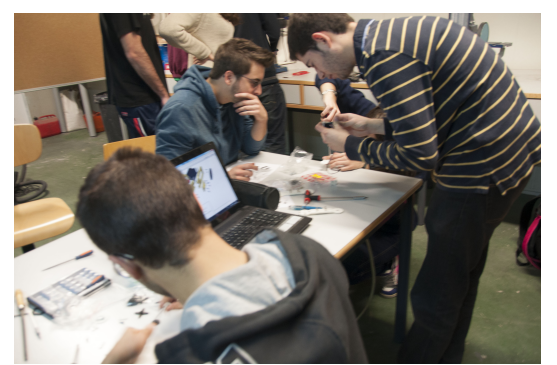

(b) Team 1

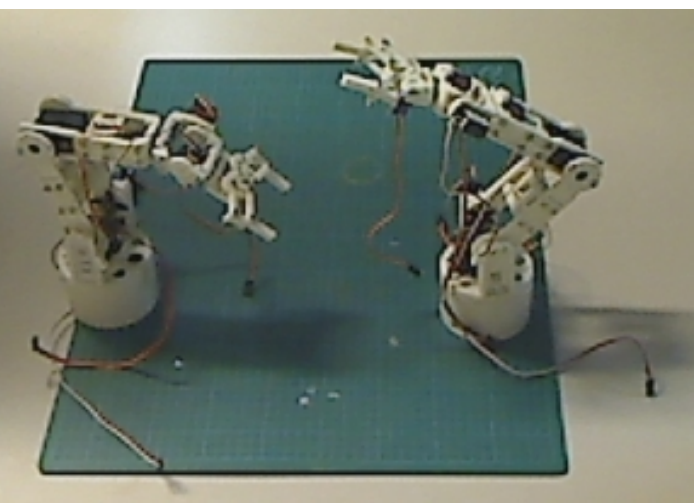

(d) Final result

Fig. 7 Drilling and assembling two robot arms.

\subsubsection{Simulation of a 6 DOF Robotic Arm}

In this section, we describe the complete process for simulating the 6 DOF Robotic Arm, including forward and inverse kinematics. Online Resource Material 4 contains a video with simulations that students are required to reproduce. The lab sessions are oriented to reinforcing all basic theory concepts rather than being a complete V-REP tutorial, so as with the Miniskybot 2 simulation described above, preliminary activities are focused on getting students familiar with V-REP by importing robot parts, positioning and rotating them, and attaching joints to links. These activities lead students to obtaining a simulated robot with an appropriate visual appearance as shown in Figure 8(a). In order to provide realistic motion, all arm joints are configured in a force/torque mode, which implies that dynamic aspects are also taken into account. The joints of the grip, shown in Figure 8(b), are treated in a different manner, because none of them is motorized except the one controlling the grip. These joints must be configured in inverse kinematic mode, abstracting from the complexities behind closed-kinematic chains (V-REP computes necessary joint angles to ensure the chain remains closed). The joint associated with the gear mechanism is configured in dependent mode (i.e., it mimics the angle of 
the motorized gear opposite). A set of dynamic and collidable objects is created and configured, placed on the hidden layers as shown in Figure 8(c), and attached to joints.

The Denavit-Hartenberg (DH) method [8] is one of the fundamental aspects that students learn during the course. They are required to obtain a table with four DH parameters for each joint. After that, they attach to each link a reference frame that they need to place based on the DH method. From the DH parameters, they obtain transformation matrices relating each reference frame and set them accordingly using V-REP API as shown in Figure 8(d). As a result, reference frames are placed on with their appropriate positions and orientated correctly as can be seen in Figure 8(e).

In order to solve the inverse kinematic problem, two different approaches are followed: in the first, students use a geometric approach to find the exact solution for this particular robot arm. In the second, they use a V-REP inverse kinematic module to compute the arm angles. The purpose behind this is that students understand the differences between exact inverse kinematics and approximate inverse kinematic methods [34].

Remaining lab sessions are focused on understanding other basic concepts in V-REP such as user interfaces, graphs and paths. To complete their initial training, students are required to implement a pick and place application (see Figure $8(\mathrm{~g})$ ) using V-REP functionalities.

\subsection{Bachelor Degree and Master Thesis Final Projects}

The Robots shown in Figure 1, and in particular the ones described in previous sections of this paper, represent just some examples of different types of robots. Our purpose is to replicate and/or adapt them progressively (depending to some extent on students' needs) as well as improve specific aspects over time. Below are some brief descriptions of how robots have been used recently in students Bachelor Degree Final Projects (FP) and Master theses.

For instance, the ROFI robot [9] in Figure 9(a) was printed, assembled and adapted as part of a student's FP. Figure 9(a) shows the final result, where both legs have been printed and validated according to the original design while a new body with arms has replaced the original head to make room for different electronics. Another student is simulating walking modes of ROFI in V-REP (shown in Figure 9(b)), which involves similar tasks performed with the robots described in previous sections.

In other projects, two students have designed a mobile base and associated electronics for a Robot Arm (Figure 9(c)) in order to extend its capabilities, particularly targeting pick and place with mobile manipulators, while a group of three students on our European Industrial Management course (a POL course similar to EPS) first researched a wide range of suppliers and sources on the international market to compare and select the appropriate components for a Hummingbird II AUV. Their choices were then printed and the Hummingbird II assembled (Figure 9(d)). 


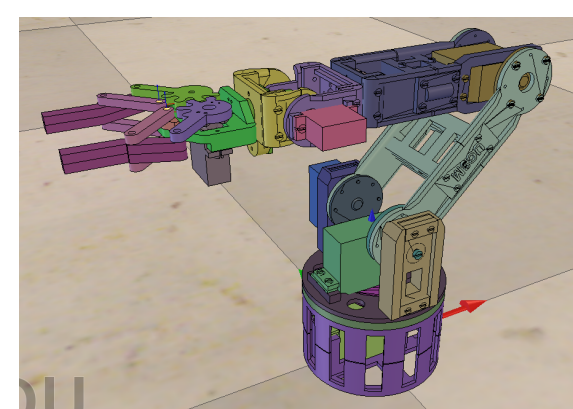

(a) Visual aspect

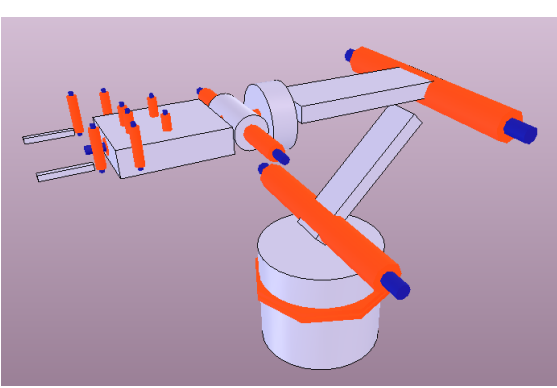

(c) Dynamic and collidable objects

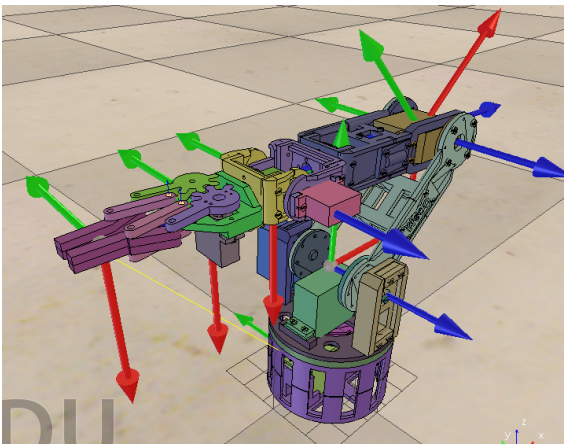

(e) Denavit-Hartenberg

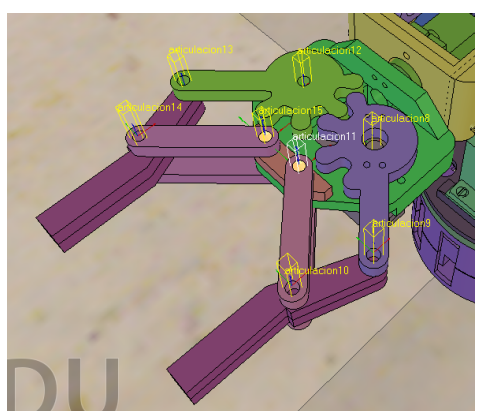

(b) Robot grip

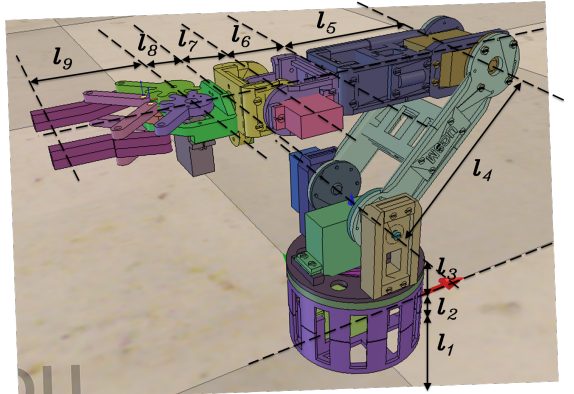

(d) Link lengths

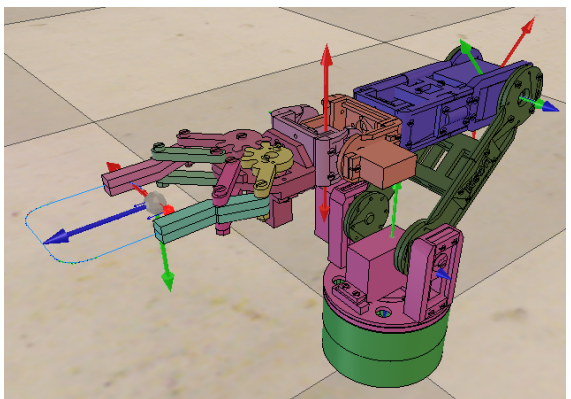

(f) Inverse kinematics

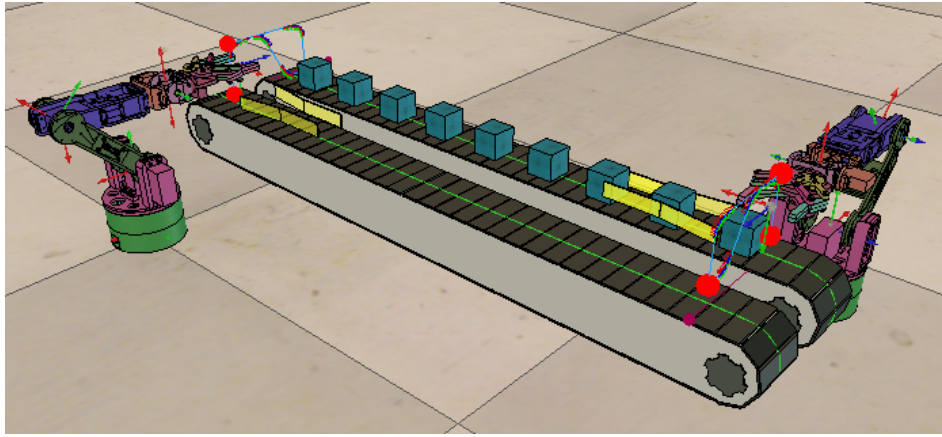

(g) Pick \& place

Fig. 8 Simulation of a robot arm with 6 DOF. 

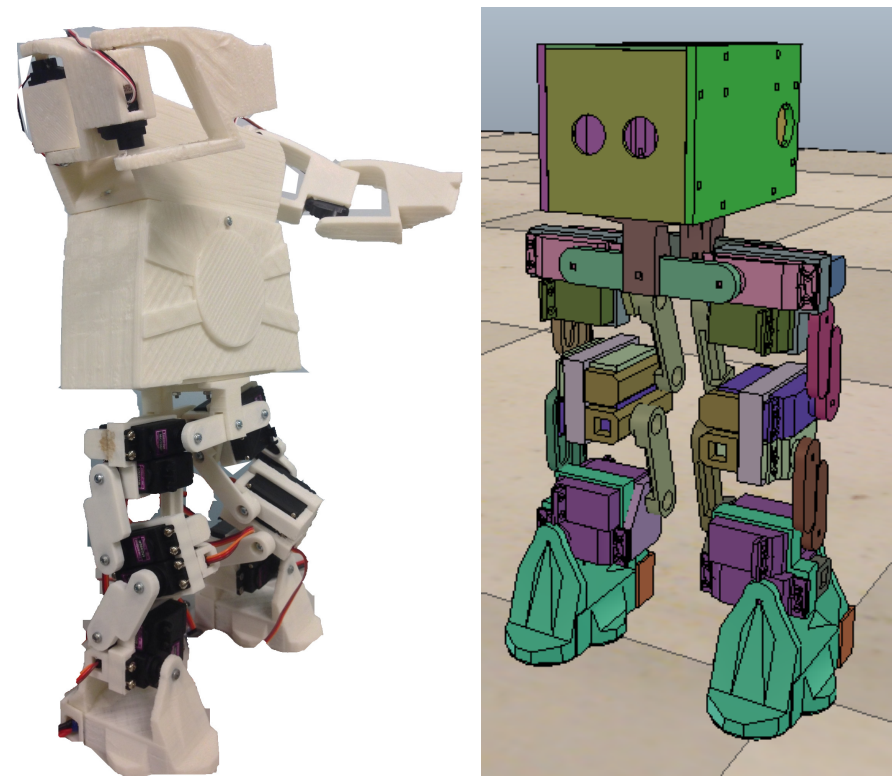

(a) Printed ROFI robot with re- (b) Simulated original ROFI designed body robot

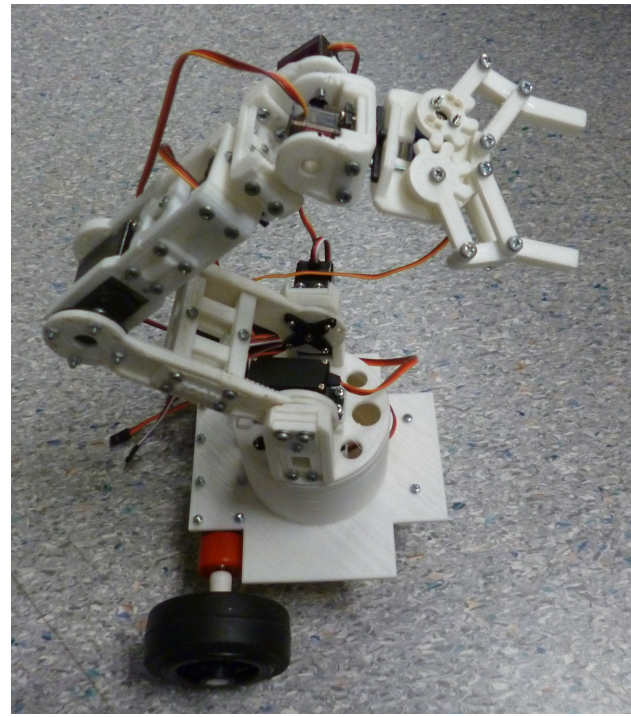

(c) Mobile Manipulator

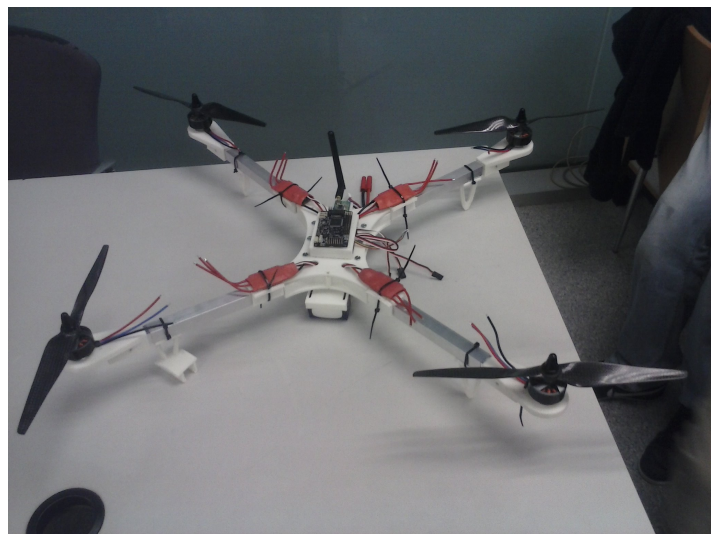

(d) Printed Hummingbird II robot

Fig. 9 Additional on-going projects. 
A further group of three students have been working with the HF08 robot. One member of the group focused on implementing visual-servoing tasks; i.e., following a specific colored object using a webcam. Another student implemented inverse kinematics for legs with Arduino. The third member of this group developed a ROS driver (Robot Operating System) to control the hexapod. Finally, a postgraduate student is using HF08 to validate reinforcement learning techniques as part of his Master thesis.

\section{Results}

This Section is divided into two parts. In the first (Section 5.1), we describe the assessment tools used to evaluate students' performance and their acquisition of competences during the European Project Semester. In addition, we provide some examples of what the students carrying out the Robotics Project during the academic year 2013/2014 said about the program. In the second (Section 5.2), we describe the results obtained from surveys of students on the Mobile Robotics course. The surveys were designed to measure the students' perceptions of specific aspects of the course as well as their perceptions regarding the acquisition of competences.

\subsection{Acquisition and evaluation of competences in EPS}

\subsubsection{Assesment Tools}

The inherent complexity of team-based activities can create ambiguity for academic staff and students in terms of learning goals and about what constitutes evidence of learning [10]. In EPS, we evaluate and assess the mindset and practical knowledge of teams and individuals. The students are involved in a complex cycle of thinking-action-feedback-thinking-action-feedback.

Oral (as opposed to written) examinations are especially useful to explore and assess design thinking, as they allow an emphasis on exploring an individual student's understanding of key decision points in the design process. Oral examinations also offer a more comprehensive method for exploring the strengths and limits of a student's technical knowledge and skills. In EPS, a board of examiners of between 3 to 5 members seeks evidence of student professionalism in their documentation and presentations. The assessment process, in fact, is designed to be primarily motivational and to encourage students to build a broad understanding of a team-based project. Written reports and oral presentations are discussed in detail with each team with a view to improving subsequent performance.

The 5 principal assessment tools we use are described below. They are based on many discussions with colleagues from our own University as well as from other Higher Education Institutions, research literature, and our own observations and reflections, thus, they are drawn from many sources. These 
include research group discussions, formal analyses of interview transcripts, presentations given by participants and colleagues at workshops and symposia and anecdotes told during informal conversations.

1. A written self-evaluation by the students focused on: a) what they believe they have contributed to the project in terms of their specialist subject matter; b) what they believe they have contributed to the process (i.e., the teamwork); c) what they believe they have contributed overall (product and process) to the work done so far.

2. Students provide peer evaluations focused on the contributions and mindset of their team members. These evaluations are carried out halfway through the program (when they can serve as a useful 'wake-up call' to those students who are not pulling their weight) and at the end. In addition, each team should agree unanimously on the distribution of 100 points among the team members. If all is going well, every team member should receive the same number of points.

3. The students provide peer assessments of the other teams (usually there are 6 or 7 EPS projects running concurrently). As part of the main public presentations of team projects, the participants are required to provide quantitative and qualitative feedback. This is valuable both to improve the communication skills of the teams and to develop the critical assessment and listening skills of the students. Assessed aspects include: planning, objectives, content, approach, organization, visual aids, delivery and language. Each of these aspects is rated on a 1 to 5 scale, and a further, global rating is also given.

4. Project supervisors complete an Evaluation Chart with 12 given parameters:

- Dynamism and motivation.

- Efficiency at work.

- The ability to develop new knowledge.

- The ability to produce an operational report (group work).

- Adaptation and integration into a team.

- Sense of observation.

- Sense of organization.

- Report writing (individual contribution).

- The ability to work out and analyse the project.

- The ability to apply appropriate knowledge.

- Self-reliance and initiative.

An overall assessment of each team member is generated from the chart. Other factors such as conscientiousness, communication skills, attendance and punctuality may also be taken into account. Supervisors' comments are intended to be constructive and encouraging.

Figure 10 shows the average supervisor assessment of the 12 parameters for the academic year 2013/2014. Each item was graded on a scale of 0 to 6 , were 0 is unacceptable, 3 is average and 6 is excellent. 


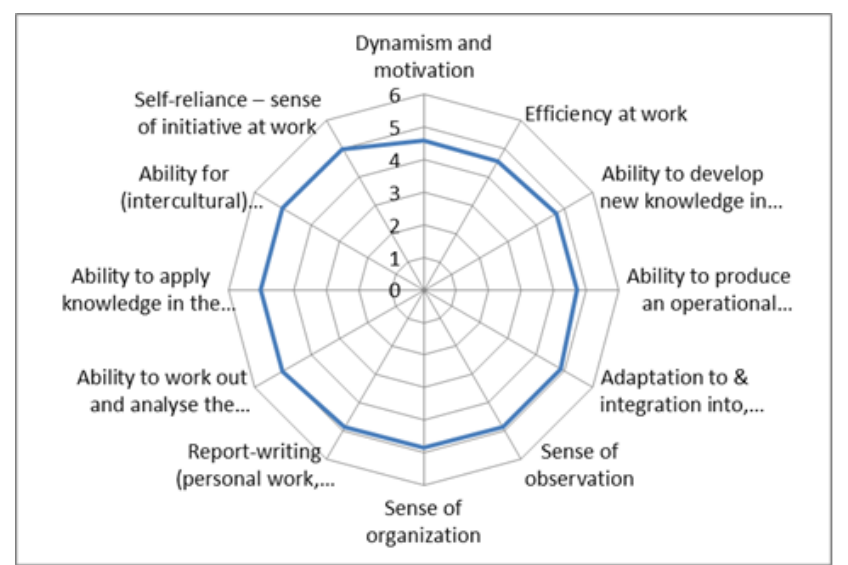

Fig. 10 Supervisors' assesment of EPS teams for the academic year 2013/2014.

5. The students are evaluated by external examiners. Academic colleagues from Spain and abroad as well as colleagues from industry participate in the overall assessment. They evaluate both oral and written presentations. External examiners consider aspects such as: specialist subject matter knowledge; the ability to work independently; organizational skills; personal initiative; the ability to integrate into the company; effective communication skills and the ability to work with others.

In addition to the above, and to better determine an individual student's contributions to their team's deliverables, EPS supervisors carry out the following periodic monitoring activities: direct observation of teams, regular supervisory meetings, specific requirements regarding presentations and documentation; the submission of team meeting minutes and the fulfillment of specific (milestone) tasks throughout the term either for individuals or for the team. Other formative assessment opportunities include written reports such as design briefs, requirements reports, status reports, presentations and, of course, the full techical report which students are required to write including plans, drawings, technical specifications and so on. In turn, supervisors produce a written assessment regarding the quality of the content of the report, its structure, layout, clarity and the appropriateness of the language.

\subsubsection{Student's feedback}

When they have completed the EPS course, all students are asked for their feedback. Their answers nearly always include remarks about how their selfesteem has improved, about their greater awareness of the importance of soft skills and about their increased tolerance and understanding of cultural differences. Some examples of the comments from students on the robotics project during EPS 2013/2014 follow. Bear in mind that these students are from many 
nationalities and their first language in most cases is not English. The comments are as written; that is, they have not been altered in any way:

- "The possibility to show the outcome of my learning period at the end was an extra motivation, and changed my attitude to the problem to solve".

- "We were very motivated because we have a clear aim, and that aim was relevant for us, and we wanted to work together to reach our aim".

- "The continuous participation in the team meetings developed our ability to solve the given prob-lem and to develop our soft skills".

- "We learned a lot from our mistakes. The trial and error method can be used efficiently with printbots and we gained a lot of confidence thanks to that".

- "Being working with printbots challenged myself and provided hands-on experience with real problems".

- "The development of a business model from printbots provides me an easier way to integrate what I have learned into wider systems".

- "I consider the printbots provide a clear opportunity to see the relationship between the practical things and the written work".

- "I increased my ability to planning my time, because I was very motivated to reach the goal. I like the responsibility to solve a realistic problem".

- "I developed my leadership skills in different situations and tried to motivated others".

- "Working in a team, means carrying responsibility for the whole team and I felt a clear development of my teamwork competences".

- "A big improvement on communication was experienced during the semester. Initially, misunderstandings were usual, but the intracommunication was very professional at the end as well as the dissemination presentations".

- "The entire group was interested and has been working hard to deliver a great quality technical report as I never seen before".

- "It was spectacular the difference on the willingness to build upon the ideas of others since the first day to the last meeting. We developed a lot our intercultural competence".

- "I found very convenient the idea to develop learning skills from basic to advanced literature, and from practical work to meeting discussions and coaching. I feel that model will be useful for the rest of my life".

- "Working with printbots we understand robotics quicker and deeper than following a traditional course, I guess".

- "This step by step approach and DIY perspective makes mandatory a high level of understanding about robotics".

- "I never seen this high level of satisfaction with the result and the experience in the framework of the University".

The final outcome of the project described in Section 3 was a well-designed and well-planned conceptual model that satisfied the standards and requirements in force at the time for this kind of product. 


\begin{tabular}{|c|c|c|c|c|c|c|c|c|}
\hline \hline Year & \multicolumn{2}{|c|}{ Course } & \multicolumn{2}{c|}{ Lecturer } & \multicolumn{2}{c|}{ Department } & \multicolumn{2}{c|}{ University } \\
\hline & Qual. & \# Surv. & Qual. & \# Surv. & Qual. & \# Surv. & Qual. & \# Surv. \\
\hline $10 / 11$ & 8.1 & 26 & 7.86 & 38 & 7.2 & 2505 & 7.25 & 173.607 \\
\hline $11 / 12$ & 7.22 & 61 & 7.32 & 76 & 7.09 & 2521 & 7.24 & 186.693 \\
\hline $12 / 13$ & 6.63 & 48 & 6.55 & 76 & 6.95 & 2277 & 6.95 & 196.110 \\
\hline $13 / 14$ & $\mathbf{8 . 0 2}$ & 34 & 6.35 & 87 & 6.73 & 2941 & 6.99 & 192.419 \\
\hline \hline
\end{tabular}

Table 1 ICE survey results for the Mobile Robotics course.

\subsection{Surveys of Robotics Courses}

Each academic year, students have the opportunity to complete a course survey in order to provide feedback. The surveys are intended to measure the students' perceptions of a lecturer's knowledge of his or her subject, the planning and organisation of the course, the methodology employed, whether and to what degree the lecturer motivates the students, and their (the students') overall satisfaction. All the questionnaires are administered anonymously by the University's own Institute of Education Science (ICE).

Here, we present the results we have obtained for the Mobile Robotics course $^{5}$, focusing on the last four academic years. Previous to 2013-2014, there was a general downward trend in student satisfaction over the whole university (with over 170,000 surveys/year). This can be clearly seen in Table 1 which shows data for the Mobile Robotics course, the lecturer, the Department and the University. The reasons behind such results might be related to the crisis in Spain, the lack of job opportunities and possibly to academic aspects such as the gradual implantation of the Bologna Process during these years. However, we can also see that the downward trend is halted in the academic year 2013/2014 when the methodology described in this paper was introduced. Students following the course in that year rated it quite highly.

In addition to this, students attending the Mobile Robotics course were asked two more specific questions. Each question was graded on a Likert scale of 1 to 5 corresponding to "completely disagree", "somewhat disagree", "cannot say", "somewhat agree", "completely agree". A total of 31 students completed the survey.

The first question referred to the acquisition of the 13 UPV transversal competencies [33]. Students were asked:

Has the course contributed to develop the following transversal competencies?

1. Understanding (and integration)

2. Applied thinking

3. Problem solving and analysis

4. Innovation, creativity and entrepreneurship

5. Project design

6. Team-work (and leadership)

${ }^{5}$ Unfortunately, we do not have enough empirical data for the Robot Manipulator Course to show valid results. 
7. Ethics

8. Effective communication

9. Critical thinking

10. Attaining knowledge of contemporary issues

11. Lifelong learning

12. Planning and time management

13. Specialized tools

Figure 11 clearly shows that for this specific course students rated 6 of the 13 competencies as "somewhat agree"; in particular those competencies numbered 1-5 and number 13. Most of the students (varying between 14 to 17) sought some kind of evidence regarding the development of such competencies. Others completely agree and others cannot say. Just one student somewhat disagrees with competency 5 and two of them somewhat disagree with competency 3 . These can be considered marginal cases because they represent less than $2 \%$ of all responses regarding these competencies (3 over $31 \times 5$ ). Competences 9, 10 and 11 are in between "cannot say" and "somewhat agree" with a mean value around 3.5 , which implies that some students saw some evidence of the development of those competences, but others cannot say (roughly $50 \%$ each). In general, students do not consider that the course has contributed enough to the remaining competencies, although "Ethics" is the only one with a mean value below 3 . Indeed the students' opinions ranged widely from completely agree to completely disagree, with the majority coinciding with "somewhat disagree". This result is to be expected as none of the coursework was designed to encourage this competency.

The second question referred to the planned activities on key factors for innovation $[2,4]$. Students were asked:

Has the planned activities (Lab sessions and working with printed robots) provided you the opportunity to improve or experience these aspects of the learning?

1. Skills as Engineers

2. Competencies

3. Problem solving

4. Applied robotics

5. Self-learning skills

6. Team-Work

7. Motivation

8. Confidence

9. Experience

In this case, students manifest overall satisfaction with their acquisition of key factors for innovation. All the aspects but one received a "somewhat agree" grade (or close to it). It is particularly interesting that "Team-work" was mainly graded with "cannot say", but this might be explained by the fact that in all the lab exercises and the assignment with the Miniskybot2 robot, the teams were limited to two students. They did not feel like a team but as colleagues with a shared task to do. Apart from this rather idiosyncratic 


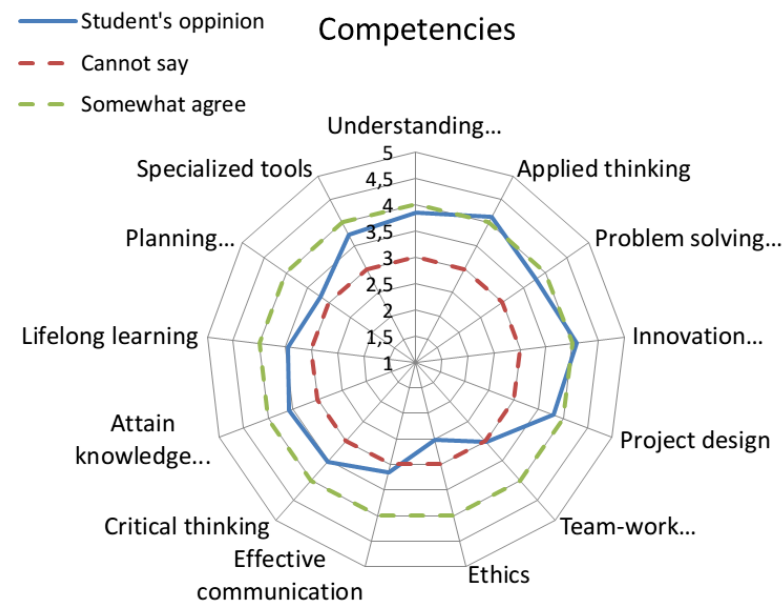

(a) Question 1

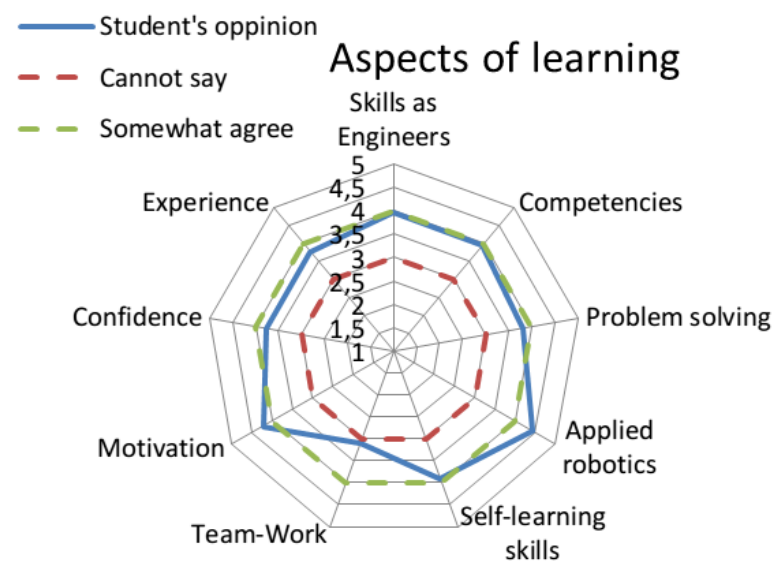

(b) Question 2

Fig. 11 Survey conducted with 31 students

result, students have positively graded the introduction of printed robots together with simulation tools as interesting activities to learn from practice and experience.

\section{Conclusions}

In response to a call for innovative models of education from industry, government and students, this paper presented some ways of incorporating printbots into conventional robotics courses or project work and using them as highly 
stimulating teaching/learning resources. These ideas have been gathered over nearly a decade of working with students from different academic backgrounds and nationalities. In general terms we can say that printable robots offer an excellent basis for teaching a number of different engineering disciplines and facilitate the acquisition of knowledge and its application to solving real problems. Some of the more specific educational benefits include:

- Encouraging the development of skills, competences and attitudes to solve a given problem or set of problems in realistic contexts

- Giving students experience in understanding and implementing robotics principles from primary research literature

- Strengthening understanding

- Enhancing lifelong learning skills

- Fostering teamwork and collaboration (in EPS)

- Raising students' awareness of the need for writing up good technical reports (in EPS)

In addition students:

- clearly see the relationship between the practical and the written work

- gain hands-on experience with real problems

- gain confidence in their own abilities

- are more motivated

- experience higher levels of satisfaction

- find it easier to integrate what they have learned into wider systems

We have shown that the ideas presented here are educationally sound and can be used as plausible pedagogic alternatives in the field of Robotics. The ideas and methods discussed in this paper can be easily adapted to other university syllabi and, with appropriate adaptation of program and contents, also to other educational levels. In our opinion, due to the increasing availability of a large number of free printbots and open source platforms, the Robotics Community now has the opportunity to take a great leap forward.

Acknowledgements The authors would like to thank Miguel Fernandez, Andrés Conejero and Vicente Franch for their kind support in 3D printing, drilling and assembling parts, as well as all the students who have participated in the various stages of this work. We also would like to thank to the School of Design at UPV and particularly to Enrique Ballester, the Head of the School.

\section{References}

1. Criteria for accrediting engineering programs. 2014-2015, 2014-2015. http://www . abet . org/eac-criteria-2014-2015.

2. N. S. Board. Moving forward to improve engineering education, 2007. http://www.nsf . gov/pubs/2007/nsb07122/nsb07122.pdf.

3. G. Campion, G. Bastin, and B. d'Andréa Novel. Structural properties and classification of kinematic and dynamic models of wheeled mobile robots. IEEE Transactions on Robotics and Automation, 12(1):47-62, 1996. 
4. A. R. Carberry, H.-S. Lee, and M. W. Ohland. Measuring engineering design self-efficacy. Journal of Engineering Education, 99(1):71-79, 2010.

5. A. Castro. Robotic arm with 6 dof, 2012. http://www.thingiverse.com/thing: 30163 .

6. H. Choset, K. M. Lynch, S. Hutchinson, G. A. Kantor, W. Burgard, L. E. Kavraki, and S. Thrun. Principles of Robot Motion: Theory, Algorithms, and Implementations. MIT Press, Cambridge, MA, June 2005.

7. B. d'Andréa Novel, G. Campion, and G. Bastin. Control of nonholonomic wheeled mobile robots by state feedback linearization. International Journal on Robotics Research, 14(6):543-559, 1995.

8. J. Denavit and R. S. Hartenberg. A kinematic notation for lower-pair mechanisms based on matrices. Trans ASME J. Appl. Mech, 22(2):215221, 1955.

9. J. Dowdall. Rofi robot five, 2012. http://www.projectbiped.com/prototypes/rofi.

10. M. Eliot, P. Howard, F. Nouwens, A. Stojcevski, L. Mann, J. Prpic, R. Gabb, S. Venkatesan, and A. Kolmos. Developing a conceptual model for the effective assessment of individual student learning in team-based subjects. Australasian Journal of Engineering Education, 18(1):105-112, 2012.

11. D. Fox, W. Burgard, and S. Thrun. The dynamic window approach to collision avoidance. Robotics Automation Magazine, IEEE, 4(1):23-33, 1997.

12. P. Fuentes-Dura, L. Armesto, and D. Perry. Multidisciplinary projects: Critical points and perceptions in valladolid in innovation and quality in engineering education. In Innovation and Quality in Engineering Education, pages 315-331, 2012.

13. P. Fuentes-Dura, M. P. Cazorla, M. G. Molina, and D. Perry. European project semester: Good practices for competence acquisition. In Valencia Global, pages 165-172, 2014.

14. J. González, A. Barrientos, A. Prieto-Moreno, and M. A. de Frutos. Miniskybot 2, 2012. http://www. iearobotics.com/wiki/index . php?title=Miniskybot_2.

15. J. Gonzalez-Gomez, A. Valero-Gomez, A. Prieto-Moreno, and M. Abderrahim. A new open source 3d-printable mobile robotic platform for education. In U. Rckert, S. Joaquin, and W. Felix, editors, Advances in Autonomous Mini Robots, pages 49-62. Springer Berlin Heidelberg, 2012.

16. J. Gonzlez and R. Wagenaar, editors. Tuning Educational Structures in Europe. University of Deusto and Groningen. Deusto, 2003.

17. E. Heinrich, M. Bhattacharya, and R. Rayudu. Preparation for lifelong learning using eportfolios. European J. Engineering Education, 32(6):653-663, 2007.

18. O. Khatib. Real-time obstacle avoidance for manipulators and mobile robots. The International Journal of Robotics Research, 5(1):90-98, 1986.

19. J. Krassman. Quadcopter hummingbird ii, 2013. http://www. thingiverse.com/thing: 167721.

20. G. Langevin. Inmoov, 2012. http://www.inmoov.fr.

21. Madox. ecanum wheel rover 2, 2011. http://www.madox.net/blog/2011/01/24/ mecanum-wheel-rover- 2 .

22. M. B. Miles and A. M. Huberman. Qualitative Data Analysis: An Expanded Sourcebook. SAGE Publications, 1994.

23. J. Minguez and L. Montano. Nearness diagram (nd) navigation: Collision avoidance in troublesome scenarios. IEEE Transactions on Robotics and Automation, 20:2004, 2004.

24. Olalla. Caterpillator v1.1, 2011. http://www.thingiverse.com/thing:8559.

25. A. Ollero. Robótica. Manipuladores y robots móviles. Marcombo, S.A., Barcelona, 2001

26. M. Price. Hf08 hexapod robot, 2012. http://www.heliumfrog.com/hf08robot/ hf08blog.html.

27. K. Rawat and G. Massiha. A hands-on laboratory based approach to undergraduate robotics education. In Proceedings of 2004 IEEE International Conference on Robotics and Automation, volume 2, page 13701374, 2004.

28. C. Robotics. Virtual experimentation robotic platform (v-rep), 2013. www . coppeliarobotics.com.

29. B. Scott. Principles of problem and project based learning the aalborg model. Aalbord University, 2010.

30. U. Teichler and H. Schonburg, editors. Comparative Perspectives on Higher Education and Graduate Employment and Work Experiences from Twelve Countries. Kluwer Pub., 2004. 
31. I. Ulrich and J. Borenstein. Vfh+: reliable obstacle avoidance for fast mobile robots. In Robotics and Automation, 1998. Proceedings, volume 2, pages 1572-1577, 1998.

32. I. Verner, S. Waks, and E. Kolberg. Educational robotics: An insight into systems engineering. European Journal of Engineering Education, 24(2):201-212, 1999.

33. C. y. A. Vicerrectorado de Estudios. Dimensiones competenciales upv, 2013. http: //www .upv.es/contenidos/ICEP/info/DimensionesCompetenciales.pdf.

34. C. W. Wampler. Manipulator inverse kinematic solutions based on vector formulations and damped least squares methods. IEEE Transactions on Systems, Man, and Cybernetics, 16(1):93-101, 1986.

35. J. Weinberg and X. Yu. Robotics in education: Low-cost platforms for teaching integrated systems. Robotics Automation Magazine, IEEE, 10(2):4-6, June 2003. 